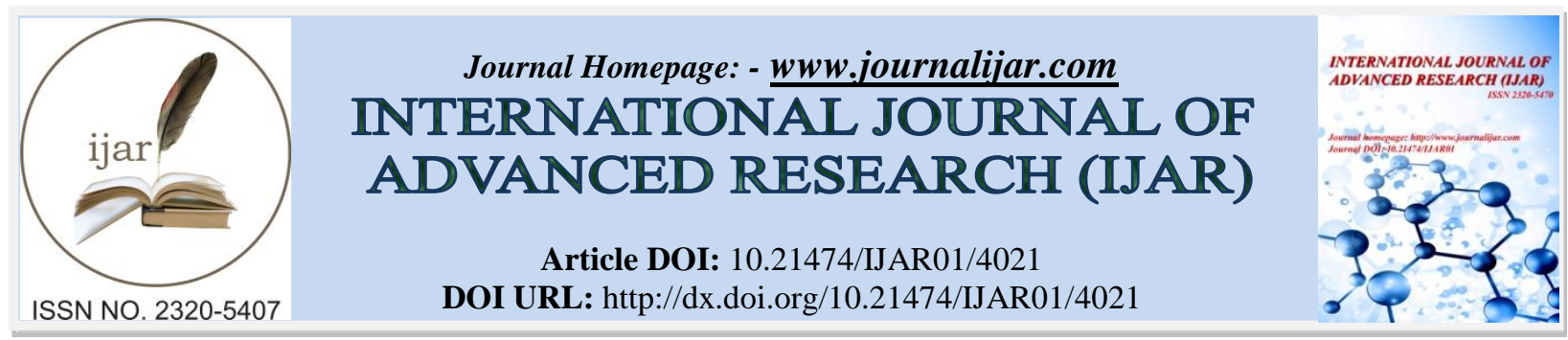

RESEARCH ARTICLE

\title{
HEART-TYPE FATTY ACID-BINDING PROTEIN (H-FABP) IS A MORE ACCURATE BIOMARKER FOR EARLY DIAGNOSIS OF PATIENTS WITH ACUTE CORONARY SYNDROME.
}

Mohamed Eissa $^{1}$, Ekhlas Hussein ${ }^{2}$, Zizi Saad $^{2}$ and Mohammad Elrawy ${ }^{2}$.

1. Clinical Pathology Department, Faculty of Medicine, Zagazig University and Pathology Department College of Medicine, King Khalid University.

2. Cardiology Department, Faculty of Medicine, Zagazig University, Egypt.

\section{Manuscript Info}

Manuscript History

Received: 24 February 2017

Final Accepted: 20 March 2017

Published: April 2017

Key words:-

Heart-type Fatty Acid-Binding Protein,

LDH-1, MYO, CK-MB, cTnT and acute

coronary syndrome

\section{Abstract}

Background: Old biochemical markers were used as acute myocardial infarction (AMI) indicators such as serum Aspartate Transaminase (AST) and Lactic Dehydrogenase-1 (LDH-1), Myoglobin (MYO), Creatine Kinase-MB (CK-MB), Troponin T and I (cTn-T, cTn-I). Later, other cardiac biomarkers such as H-FABP can be detected in the blood within one to three hours of the pain.

Aim of the work: To determine the efficacy of H-FABP compared with cTn-T as an early biomarker in the acute coronary syndrome with ST segment elevation myocardial infarction (ACS-STEMI) in patients presenting with acute cardiac chest pain.

Patients and methods: This work was carried out on 75 patients fulfilled the inclusion criteria and 25 subjects matched for age and risk factors were included as a control group. All of the patients and controls were subjected to routine clinical, laboratory investigations and assay of cardiac enzymes (cTn-T and h-FABP).

Results: This study showed that H-FABP was a sensitive and specific marker for the early detection of AMI, especially within $3 \mathrm{~h}$ after the onset of chest pain $(92.86 \%$ and $90.0 \%$ versus $81.8 \%$ and $88.2 \%$ for Troponin), with high diagnostic accuracy for AMI in 0-3 h from onset of chest pain than Troponin (92.45 versus 85.7 for Troponin).

Conclusion: H-FABP was an early marker of ischemia; that was detected before any detectable change in (cTn-T) even in the absence of myocardial necrosis.

Copy Right, IJAR, 2017,. All rights reserved.

\section{Introduction:-}

Old biochemical markers were used as acute myocardial infarction (AMI) indicators such as serum aspartate transaminase (AST) and lactic dehydrogenase-1 (LDH-1). Myoglobin (MYO), creatine kinase-MB (CK-MB), troponin $\mathrm{T}$ and I (cTn-T, cTn-I) are widely used in diagnosing patients with acute chest pain for the detection of myocardial tissue damage and also manage high-risk patients. However, they do not possess high diagnostic sensitivity or specificity for AMI in early stage, especially within 3 hours after onset of persistent angina pectoris. (1) 
MYO is more sensitive in the early phase of AMI, but its specificity is not satisfactory. Heart-type Fatty AcidBinding Protein (H-FABP) is a marker of myocardial ischemia even in the absence of frank necrosis, and may be a useful tool as prognostic value of elevated H-FABP for monitoring of a recurrent infarction. (2)

Later, other cardiac biomarkers such as glycogen phosphorylase BB (GP-BB) and carbonic anhydrase III (CA-III) were assessed. (3)

Forty percent of patients presenting with acute chest pain could be spared the risks and costs of unnecessary hospital admissions and more invasive cardiac testing by simply adding test to the diagnostic protocol to rule out heart attack. (4)

H-FABP can be detected in the blood within one to three hours of the pain and is 20 times more specific to cardiac muscle than myoglobin. (5)

H-FABP is a more sensitive biomarker for myocardial infarction than troponin at 3-6 hours following chest pain onset. This sensitivity may be explained by the high concentration of H-FABP in myocardium compared to other tissues, the stability and solubility of H-FABP, its low molecular weight compared to MYO, CK-MB and cTnT respectively. (6)

\section{Aim of the work:-}

To determine the efficacy of the use of heart-type fatty acid binding protein (H-FABP) compared with troponin $(\mathrm{cTn}-\mathrm{T})$ as a novel biomarker in the acute coronary syndrome with ST segment elevation myocardial infarction (ACS-STEMI).

\section{c and Methods:-}

This work was carried out on 75 patients fulfilled the inclusion criteria and 25 subjects matched for age and risk factors were included as a control group; making the whole study population 100 subjects from Zagazig University Hospital emergency department (ED) and National Heart Institute (NHI) cardiac chest pain units aiming to determine the efficacy of heart-type fatty acid binding protein (H-FABP) as a novel biomarker in the early diagnosis of acute coronary syndrome (ACS-STEMI) in patients presenting with acute cardiac chest pain during the period between December 2013 up to May 2014. Institutional Review Board (IRB) approved the study by ethical committee in the faculty of medicine and from patients included in the study.

\section{Inclusion Criteria:-}

$>$ Age: any age.

$>$ Sex: both males and females.

$>$ Diagnosis: patients admitted with ACS-STEMI and accepted to participate in this study.

\section{Exclusion criteria:-}

All patients who refused to give an informed consent to participate in this study as well as if the symptom onset of chest pain was more than 6 hours were excluded from the study. All causes that may result in cardiac biomarker elevation in absence of ACS have been also excluded from study as:

$>$ Right ventricular (RV) dysfunction

$>$ Radiofrequency catheter ablation within 12 hours

$>$ PCI (Primary Cutaneous Intervention) or coronary artery bypass graft (CABG) within 30 days

$>$ Prior AMI within 30 days

$>$ Patients who received direct current (DCC) shock

$>$ Significant renal insufficiency $(\mathrm{Cr} \mathrm{Cl}<60 \mathrm{ml} / \mathrm{min})$

$>$ Recent cardiac surgery

$>$ Chronic muscle disease

$>$ Pregnancy

$>$ Currently receiving corticosteroid therapy or anabolic steroids

$>$ Collagen diseases 


\section{All patients were subjected to:-}

1. Informed consent was taken from the patients or their relatives before clinical examination.

2. Assessment including :

$>$ Age, sex, address, marital status, special habits and family history of premature Coronary Artery Diseases (CAD) risk factors.

$>$ Complete history taking to document the presence of associated risk factors including diabetes mellitus, hypertension and dyslipidaemia.

$>$ Medications received.

3. Physical assessment including:

$>$ General examination (general look, vital signs [blood pressure, heart rate, respiratory rate and temperature] and general systemic examination).

$>$ Local cardiac examination.

Patients were ranked by Killip class in the following way:

$>$ Killip class I: individuals with no clinical signs of heart failure.

$>$ Killip class II: individuals with rales or crackles in the lungs, an $S_{3}$, and elevated jugular venous pressure.

$>$ Killip class III: individuals with frank acute pulmonary edema.

$>$ Killip class IV: individuals in cardiogenic shock or hypotension

(Measured as systolic blood pressure lower than $90 \mathrm{mmHg}$ with evidence of peripheral vasoconstriction, oliguria, and cyanosis or sweating).

\section{Investigations Including:-}

Routine laboratory investigations including:

$>$ Complete blood count (CBC; total \& differential).

$>$ Random blood sugar (RBS).

$>$ Liver enzymes (SGOT, SGPT).

$>$ Coagulation profile (PT, PTT, and INR).

$>$ Renal function tests (urea, creatinine).

$>$ Lipid profile.

$>$ Cardiac enzymes [troponin T (cTn-T), heart-type fatty acid binding protein (H-FABP)].

Human Heart Fatty Acid Binding Protein (h-FABP) Kit

Only one venous blood sample $(10 \mathrm{ml})$ was withdrawn by sterile venous puncture and clean dry syringes from the cubital vein of every investigated subject at the time of admission to the hospital within $6 \mathrm{~h}$ after the onset of symptoms.

This kit allows for the in-vitro qualitative determination of human H-FABP by instrument (QuickSens ${ }^{\circledR} \Omega 100$ ).

\section{Storage:-}

1. Unopened test kits should be stored at $2-8^{\circ} \mathrm{C}$ upon receipt and the microtiter plate was kept in a sealed bag. The test kit may be used throughout the expiration date of the kit.

2. Opened test kits will remain stable until the expiring date shown, provided it is stored as prescribed above.

\section{Electrocardiogram (ECG):-}

12-lead resting ECG was done for every patient at a paper speed of 25 milliseconds and calibrated correctly to study $\mathrm{p}$ wave, QRS complex, T wave and segmental changes (P-R and S-T segments) at presentation and to determine site of infarction by distribution of S-T segment elevation in the affected ECG leads.

\section{Conventional echocardiography:-}

Complete transthoracic echocardiography study was performed for every patient in the supine and lateral position using General Electric system Vivid-3 machine, assessing global systolic function, diastolic function, resting regional wall motion abnormalities, mechanical complications of AMI and RV function using TAPSE method. 


\section{Results:-}

Descriptive data of the studied population:-

Demographic characteristics and CAD risk factors of the studied groups:-

- The study showed a male preponderance representing (67\%) of the study population. (Table $\mathbf{1})$

- There were no statistically significant differences as regard age and gender among the studied groups. (Table $\mathbf{1}$ )

- Coronary artery disease (CAD) risk factors were relatively frequent in the studied population, DM was present in (44\%), hypertension in (50.7\%), current smokers were in (56\%), dyslipidemia were in $(38.7 \%)$ and a family history of premature CAD was present in (24\%) of the cases group. (Table 1 )

- There was a highly statistically significant difference regarding CAD risk factors; serum total cholesterol (TC), Triglycerides (TG), low density lipoprotein cholesterol (LDL-C) and high density lipoprotein cholesterol (HDLC) between the studied groups.(Table 1)

- Both systolic and diastolic blood pressure and heart rate were statistically significantly higher in case group of patients than those of control group. (Table 2)

Table 1:- Comparison between the two studied groups according to demographic data and CAD risk factors.

\begin{tabular}{|c|c|c|c|c|c|c|}
\hline & \multicolumn{2}{|c|}{$\begin{array}{l}\text { Cases } \\
(n=75)\end{array}$} & \multicolumn{2}{|c|}{$\begin{array}{l}\text { Control } \\
(\mathbf{n}=25)\end{array}$} & \multirow[t]{2}{*}{ Test of sig. } & \multirow[t]{2}{*}{$\mathbf{P}$} \\
\hline & No. & $\%$ & No. & $\%$ & & \\
\hline \multicolumn{7}{|c|}{ I-Demographic criteria as regards gender and age } \\
\hline Male & 52 & 69.3 & 15 & 60.0 & \multirow{2}{*}{$\chi^{2}=0.739$} & \multirow{2}{*}{0.390} \\
\hline Female & 23 & 30.7 & 10 & 40.0 & & \\
\hline $\begin{array}{l}\text { Age } \\
(\text { Mean } \pm \text { SD) }\end{array}$ & \multicolumn{2}{|c|}{$56.25 \pm 11.67$} & \multicolumn{2}{|c|}{$60.76 \pm 9.11$} & $\mathrm{t}=1.758$ & 0.082 \\
\hline \multicolumn{7}{|c|}{ II-Frequencies of CAD risk factors } \\
\hline \multicolumn{7}{|l|}{ Risk factor } \\
\hline $\begin{array}{l}\text { DM } \\
\text { No } \\
\text { Yes }\end{array}$ & $\begin{array}{l}42 \\
33\end{array}$ & $\begin{array}{l}56.0 \\
44.0\end{array}$ & $\begin{array}{l}25 \\
0\end{array}$ & $\begin{array}{l}100.0 \\
0.0\end{array}$ & $\chi^{2}=16.418^{*}$ & $<0.001 *$ \\
\hline $\begin{array}{c}\text { HTN } \\
\text { No } \\
\text { Yes }\end{array}$ & $\begin{array}{l}37 \\
38\end{array}$ & $\begin{array}{l}49.3 \\
50.7\end{array}$ & $\begin{array}{l}25 \\
0\end{array}$ & $\begin{array}{l}100.0 \\
0.0\end{array}$ & $\chi^{2}=20.430^{*}$ & $<0.001 *$ \\
\hline $\begin{array}{l}\text { Smoker } \\
\text { No } \\
\text { Yes } \\
\text { Ex-smoker }\end{array}$ & $\begin{array}{l}33 \\
42 \\
0\end{array}$ & $\begin{array}{l}44.0 \\
56.0 \\
0.0\end{array}$ & $\begin{array}{l}20 \\
4 \\
1\end{array}$ & $\begin{array}{l}80.0 \\
16.0 \\
4.0\end{array}$ & $\chi^{2}=13.615^{*}$ & ${ }^{\mathrm{MC}} \mathrm{p}=0.001^{*}$ \\
\hline $\begin{array}{l}\text { FH } \\
\quad-v e \\
+v e\end{array}$ & $\begin{array}{l}57 \\
18\end{array}$ & $\begin{array}{l}76.0 \\
24.0\end{array}$ & $\begin{array}{l}25 \\
0\end{array}$ & $\begin{array}{l}100.0 \\
0.0\end{array}$ & $\chi^{2}=14.282^{*}$ & $0.014^{*}$ \\
\hline $\begin{array}{l}\text { Dyslipidemia } \\
\text { No } \\
\text { Yes }\end{array}$ & $\begin{array}{l}46 \\
29\end{array}$ & $\begin{array}{l}61.3 \\
38.7\end{array}$ & $\begin{array}{l}25 \\
0\end{array}$ & $\begin{array}{l}100.0 \\
0.0\end{array}$ & $\chi^{2}=7.317^{*}$ & $<0.001 *$ \\
\hline \multicolumn{7}{|c|}{ III- Lipid profile among the case and control groups: } \\
\hline Total cholesterol & \multicolumn{2}{|c|}{$216.35 \pm 65.17$} & \multicolumn{2}{|c|}{$173.40 \pm 16.59$} & $5.222^{*}$ & $<0.001 *$ \\
\hline TG & \multicolumn{2}{|c|}{$170.99 \pm 73.65$} & \multicolumn{2}{|c|}{$109.72 \pm 25.47$} & $6.180^{*}$ & $<0.001 *$ \\
\hline HDL & \multicolumn{2}{|c|}{$41.35 \pm 13.78$} & \multicolumn{2}{|c|}{$63.36 \pm 10.13$} & $7.339^{*}$ & $<0.001^{*}$ \\
\hline $\mathbf{L D L}$ & \multicolumn{2}{|c|}{$133.71 \pm 30.20$} & \multicolumn{2}{|c|}{$101.12 \pm 17.33$} & $4.594^{*}$ & $<0.001^{*}$ \\
\hline
\end{tabular}

$\chi^{2}$ : Chi square test,

t: Student t-test

*: Statistically significant at $\mathrm{p} \leq 0.05$ 
Table 2:- Comparison between the two studied groups according to vital signs on admission.

\begin{tabular}{|l|l|l|l|l|}
\hline Vital Signs & $\begin{array}{l}\text { Cases } \\
(\mathbf{n = 7 4})\end{array}$ & $\begin{array}{l}\text { Control } \\
(\mathbf{n = 2 5})\end{array}$ & $\mathbf{T}$ & $\mathbf{P}$ \\
\hline $\begin{array}{c}\text { Systolic BP } \\
\text { Mean } \pm \text { SD. }\end{array}$ & $125.34 \pm 26.80$ & $113.0 \pm 14.58$ & $2.892^{*}$ & $0.005^{*}$ \\
\hline $\begin{array}{c}\text { Diastolic BP } \\
\text { Mean } \pm \text { SD. }\end{array}$ & $76.89 \pm 15.03$ & $70.0 \pm 9.68$ & $2.643^{*}$ & $0.010^{*}$ \\
\hline $\begin{array}{c}\text { Heart rate (HR) } \\
\text { Mean } \pm \text { SD. }\end{array}$ & $86.86 \pm 21.98$ & $76.16 \pm 10.74$ & $2.337^{*}$ & $0.022^{*}$ \\
\hline
\end{tabular}

t: Student t-test

*: Statistically significant at $\mathrm{p} \leq 0.05$

Clinical outcome among patient group during hospital Course:-

- The most common Killip class on admission was class I (38\%), whereas class IV (cardiogenic shock) was the least common class $(4 \%)$. (Table 3 )

- Arrhythmic complications constituted $28 \%$ among patient group during hospitalization; the most common dysrhythmia occurred was atrial fibrillation (5.33\%) and complete heart block (5.33\%). (Table 3)

- As regards mechanical complications; $4 \%$ patients had mechanical complications i.e. one of them had rupture anterior papillary muscle with severe mitral regurgitation and the others had ventricular septal rupture (basal septal rupture with inferior STEMI and mid-apical septal rupture with anterior STEMI). All of them belonged to H-FABP positive group of patients. (Table $\mathbf{3}$ )

- Ischemic attacks during hospital course occurred in $36 \%$ of patients in the form of re-infarction, postmyocardial angina and post-revascularization angina. (Table $\mathbf{3}$ )

- Four patients died during hospital course after their diagnosis and fulfilling aim of our study. All patients who died were of free fatty acid binding protein (H-FABP) positive group. All of them belonged to H-FABP positive group of patients. (Table 3 )

Table 3:- Comparison among patient group as regards clinical outcome during hospital course.

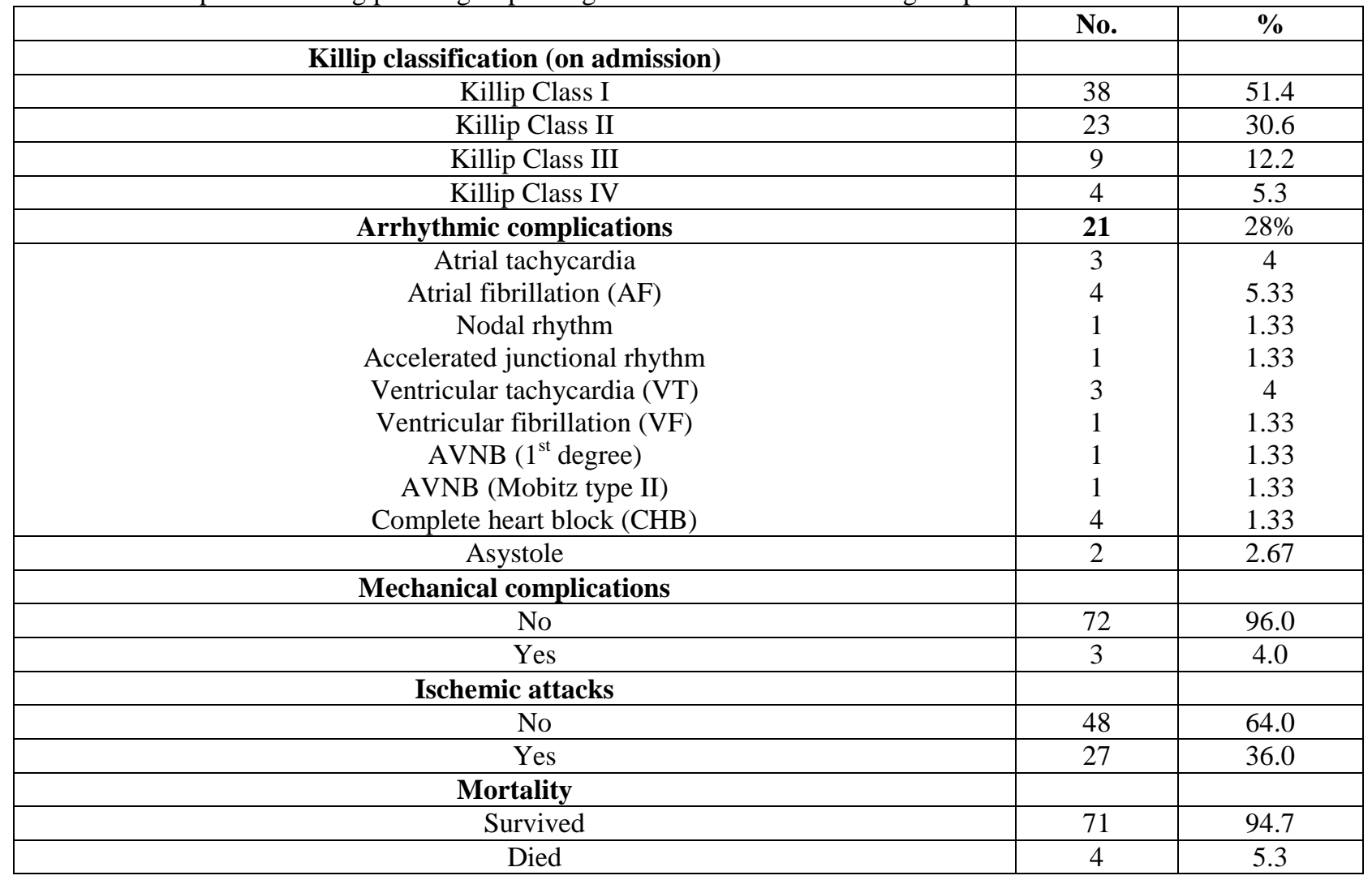


Working diagnoses among the patient group as regards site of infarction by ECG:-

- The most common working diagnoses causing the subjects in the patients group to seek medical advice in the (ED) were inferior ST segment elevation myocardial anterior (STEMI) and infarction ST segment elevation myocardial infarction STEMI (53.3\% and 40\%) respectively. (Table 4)

- The most common group of the studied population as regards of onset of chest pain 3-6 hours was (62.7\%) and those presented 0-3 hours of chest pain was (37.3\%). (Table 4)

Table 4:- Distribution of patients according to site of infarction by ECG and Onset of chest pain (h) on admission

\begin{tabular}{|c|c|c|}
\hline & No. & \% \\
\hline Site of infarction (ECG) & & $\mathbf{5 3 . 3}$ \\
\hline Anterior STEMI & $\mathbf{4 0}$ & 32.0 \\
\hline Anterior STEMI & 24 & 18.7 \\
\hline Antero-lateral STEMI & 14 & 2.6 \\
\hline Anteroseptal STEMI & 2 & $\mathbf{4 0}$ \\
\hline Inferior STEMI & $\mathbf{3 0}$ & 36 \\
\hline Inferior STEMI & 27 & 2.7 \\
\hline Infero-lateral STEMI & 2 & 1.3 \\
\hline Infero-posterior STEMI & 1 & $\mathbf{6 . 6}$ \\
\hline Lateral STEMI & $\mathbf{5}$ & 1.3 \\
\hline Lateral STEMI & 1 & 5.3 \\
\hline High lateral STEMI & 4 & 37.3 \\
\hline Onset of chest pain (h) & 28 & 62.7 \\
\hline $0<3$ & 47 & \\
\hline $3-6$ & \multicolumn{2}{|c|}{$3.35 \pm 1.75$} \\
\hline
\end{tabular}

Comparison between the case and control groups as regards troponin (cTn-I) and H-FABP:-

- As regard serum cardiac troponin T (cTn-T); there was a statistical significant difference between the 2 groups; as they were negative in all the control subjects upon presentation thereby confirming the diagnosis of MI. (Table 5)

- As regard serum heart type free fatty acid binding protein (H-FABP), there was a highly statistical significant difference between cases and controls as regard (H-FABP) qualitative results, where cases showed a higher positive results for (H-FABP) patients compared to controls subjects $(94.76 \%$ vs. 5.33\%; $\mathrm{P}$ value $=<0.001)$. (Table 5)

Table 5:- Comparison between the two studied groups according to cardiac biomarkers.

\begin{tabular}{|c|c|c|c|c|c|c|}
\hline \multirow[t]{2}{*}{ Lab investigation } & \multicolumn{2}{|c|}{$\begin{array}{l}\text { Cases } \\
(n=75)\end{array}$} & \multicolumn{2}{|c|}{$\begin{array}{l}\text { Control } \\
(\mathrm{n}=25)\end{array}$} & \multirow[t]{2}{*}{$\mathbf{T}$} & \multirow[t]{2}{*}{$\mathbf{P}$} \\
\hline & No. & $\%$ & No. & $\%$ & & \\
\hline \multicolumn{7}{|l|}{ H-FABP } \\
\hline Negative & 4 & 5.33 & 23 & 92.0 & \multirow[t]{2}{*}{$61.016^{*}$} & \multirow[t]{2}{*}{$<0.001$} \\
\hline Positive & 71 & 94.67 & 2 & 8.0 & & \\
\hline \multicolumn{7}{|l|}{ Troponin } \\
\hline Negative & 29 & 38.67 & 25 & 100.0 & \multirow[t]{2}{*}{$59.259^{*}$} & \multirow[t]{2}{*}{$<0.001$} \\
\hline Positive & 46 & 61.33 & 0 & 0.0 & & \\
\hline
\end{tabular}

$\chi^{2}$ : Chi square test

*: Statistically significant at $\mathrm{p} \leq 0.05$

Global systolic left ventricular function assessed by LVEF and WMSI among the case and control groups:-

- There was a statistically significant difference as regard EF\% and WMSI among the studied groups (P value < 0.001). (Table 6)

- $\quad$ Patients with impaired LV systolic function assessed by LVEF<40\% predominated in cases group (14.9\%) versus $(0.0 \%)$ for control group. (Table 6) 
Table 6:- Comparison between the two studied groups according to echocardiographic findings.

\begin{tabular}{|c|c|c|c|c|c|c|}
\hline & \multicolumn{2}{|c|}{$\begin{array}{l}\text { Cases } \\
(n=74)\end{array}$} & \multicolumn{2}{|c|}{$\begin{array}{l}\text { Control } \\
(\mathbf{n}=25)\end{array}$} & \multirow[t]{2}{*}{ Test of sig. } & \multirow[t]{2}{*}{$\mathbf{P}$} \\
\hline & No. & $\%$ & No. & $\%$ & & \\
\hline \multicolumn{7}{|l|}{ EF } \\
\hline$<40 \%$ & 11 & 14.9 & 0.0 & 0.0 & \multirow[t]{2}{*}{$\chi^{2}=27.383^{*}$} & \multirow[t]{2}{*}{${ }^{\mathrm{MC}} \mathrm{p}<0.001$} \\
\hline$>40 \%$ & 63 & 85.1 & 25 & 100.0 & & \\
\hline & \multirow{2}{*}{\multicolumn{2}{|c|}{$50.11 \pm 11.03$}} & \multirow{2}{*}{\multicolumn{2}{|c|}{$64.04 \pm 6.94$}} & \multirow[t]{2}{*}{$\mathrm{t}=7.370^{*}$} & \multirow[t]{2}{*}{$<0.001^{*}$} \\
\hline Mean \pm SD & & & & & & \\
\hline $\begin{array}{l}\text { WMSI } \\
\text { Mean } \pm \text { SD }\end{array}$ & \multicolumn{2}{|c|}{$1.45 \pm 0.64$} & \multicolumn{2}{|c|}{$1.0 \pm 0.0$} & $11.448^{*}$ & $<0.001^{*}$ \\
\hline
\end{tabular}

$\chi^{2}$ : Chi square test

MC: Monte Carlo test

$\mathrm{t}$ : Student t-test

*: Statistically significant at $\mathrm{p} \leq 0.05$

H-FABP positive vs. H-FABP negative groups (Univariate Descriptive Analysis):-

Demographic characteristics and frequencies of co-morbidities and risk factors of the H-FABP positive and H-FABP negative groups :

- There was higher but not statistically significant difference as regards the mean age of the H-FABP positive patients which was $(56.34 \pm 11.89$ years $)$ and that of H-FABP negative patients which was $(55.43 \pm 10.01$ years). $(\mathrm{P}$ Value $=0.846)$. (Table 7$)$

- The study also showed no statistical significant difference as regards gender predominance where the male gender predominated in the H-FABP positive patients $(71.4 \%)$ and $(69.1 \%)$ in the H-FABP negative subgroups. $(\mathrm{P}$ Value $=1,000)$. (Table 7$)$

- There was a statistical difference between the H-FABP positive and H-FABP negative patient subgroups as regard current smoking status and DM. (Table 7)

- There was no statistical significant difference between the H-FABP positive and H-FABP negative patients groups as regard as hypertension, total cholesterol, TG, HDL and LDL and family history of premature CAD. (Table 7)

Table 7:- Comparison between the two studied groups according to demographic data and CAD risk factors.

\begin{tabular}{|c|c|c|c|c|c|c|}
\hline & \multicolumn{2}{|c|}{$\begin{array}{l}\text { H-FABP } \\
\text {-ve } \quad(\mathbf{n}=7)\end{array}$} & \multicolumn{2}{|c|}{$\begin{array}{l}\text { H-FABP } \\
+ \text { ve } \quad(n=68)\end{array}$} & \multirow[t]{2}{*}{ Test of sig. } & \multirow[t]{2}{*}{$\mathbf{P}$} \\
\hline & No. & $\%$ & No. & $\%$ & & \\
\hline \multicolumn{7}{|c|}{ I-Demographic criteria as regards gender and age } \\
\hline Male & 5 & 71.4 & 47 & 69.1 & \multirow[t]{2}{*}{$\chi^{2}=0.016$} & \multirow{2}{*}{0.390} \\
\hline Female & 2 & 28.6 & 21 & 30.9 & & \\
\hline $\begin{array}{l}\text { Age } \\
(\text { Mean } \pm \text { SD) }\end{array}$ & \multicolumn{2}{|c|}{$55.43 \pm 10.01$} & \multicolumn{2}{|c|}{$56.34 \pm 11.89$} & $\mathrm{t}=0.195$ & 0.846 \\
\hline \multicolumn{7}{|c|}{ II-Frequencies of CAD risk factors } \\
\hline \multicolumn{7}{|l|}{ Risk factor } \\
\hline DM & 2 & $28.6 \%$ & 31 & 45.6 & $\chi^{2}=0.746$ & $\mathrm{FE}_{\mathrm{p}}=0.456$ \\
\hline HTN & 4 & 57.1 & 34 & 50.0 & $\chi^{2}=0.130$ & ${ }^{\mathrm{FE}} \mathrm{p}=1.000$ \\
\hline Smoker & 5 & 71.4 & 37 & 54.4 & $\chi^{2}=0.746$ & ${ }^{\mathrm{FE}} \mathrm{p}=0.456$ \\
\hline FH & 1 & 14.3 & 17 & 25.0 & $\chi^{2}=0.399$ & ${ }^{\mathrm{FE}} \mathrm{p}=0.677$ \\
\hline Dyslipidemia & 1 & 14.3 & 28 & 41.2 & $\chi^{2}=1.935$ & ${ }^{\mathrm{FE}} \mathrm{p}=0.238$ \\
\hline \multicolumn{7}{|c|}{ III- Lipid profile among the case and control groups: } \\
\hline Total cholesterol & \multicolumn{2}{|c|}{$242.0 \pm 98.67$} & \multicolumn{2}{|c|}{$213.71 \pm 61.18$} & 1.095 & 0.277 \\
\hline TG & \multicolumn{2}{|c|}{$149.57 \pm 77.87$} & \multicolumn{2}{|c|}{$173.19 \pm 73.46$} & 0.806 & 0.423 \\
\hline HDL & \multicolumn{2}{|c|}{$41.29 \pm 15.91$} & \multicolumn{2}{|c|}{$41.36 \pm 13.86$} & 0.014 & 0.989 \\
\hline LDL & \multicolumn{2}{|c|}{$127.43 \pm 33.45$} & \multicolumn{2}{|c|}{$123.32 \pm 30.09$} & 0.340 & 0.735 \\
\hline
\end{tabular}


$\mathrm{P}: \mathrm{p}$ value for comparing between the two studied groups

$\chi^{2}$ : value for Chi square test

FE: Fisher Exact test

t: Student t-test

Clinical outcome of H-FABP positive and negative patients as regards Killip classification and its comparison with Troponin positive and negative patients as regards onset of chest pain:-

- There was a significant statistical difference as regard Killip classes between the H-FABP positive and H-FABP negative patients. $(\mathrm{P}$ value $=<0.001)$. (Table 8)

- The most common type of Killip class in H-FABP positive patients is class I (56.7\%), whereas in H-FABP negative patients is type III (71.4\%) (Table 8)

- The most common onset of presentation of H-FABP positive patients was higher in $0-3$ hours $(53.33 \%)$. (Table 9)

- The most onset of presentation of troponin positive patients was from 3-6 hours from onset of chest pain $(70.5 \%)$ and that of troponin negative patients was from 0-3 hours from onset of chest pain (100.0\%). (Table 9)

Table 8:- Relation between killip class and WMSI in H-FABP positive and negative patients.

\begin{tabular}{|c|c|c|c|c|c|c|}
\hline & \multicolumn{4}{|c|}{ H-FABP } & \multirow{3}{*}{$\chi^{2}$} & \multirow[t]{3}{*}{${ }^{M C} \mathbf{p}$} \\
\hline & \multicolumn{2}{|c|}{$\begin{array}{l}\text {-ve } \\
(n=7)\end{array}$} & \multicolumn{2}{|c|}{$\begin{array}{l}+v e \\
(n=68)\end{array}$} & & \\
\hline & No. & $\%$ & No. & $\%$ & & \\
\hline \multicolumn{7}{|c|}{ Killip classification } \\
\hline Class I & 0 & 0.0 & 38 & 55.9 & \multirow[t]{4}{*}{$17.76^{*}$} & \\
\hline Class II & 2 & 28.6 & 22 & 32.4 & & $<0.001^{*}$ \\
\hline Class III & 5 & 71.4 & 4 & 5.9 & & \\
\hline Class IV & 0 & 0.0 & 4 & 5.9 & & \\
\hline
\end{tabular}

Table 9:- Relation between H-FABP and troponin as regards Onset of chest pain (h)

\begin{tabular}{|c|c|c|c|c|c|c|c|c|c|c|}
\hline \multirow{2}{*}{\multicolumn{3}{|c|}{ Onset of chest pain (h) }} & \multicolumn{4}{|c|}{ H-FABP } & \multicolumn{4}{|c|}{ Troponin } \\
\hline & & & \multicolumn{2}{|c|}{$\begin{array}{l}-v e \\
(n=4)\end{array}$} & \multicolumn{2}{|c|}{$\begin{array}{l}+v e \\
(n=71)\end{array}$} & \multicolumn{2}{|c|}{$\begin{array}{l}-v e \\
(n=29)\end{array}$} & \multicolumn{2}{|c|}{$\begin{array}{l}+v e \\
(n=46)\end{array}$} \\
\hline & No. & $\%$ & No. & $\%$ & No. & $\%$ & No. & $\%$ & No. & $\%$ \\
\hline $0-1$ & 12 & 16.0 & 3 & 75.00 & 10 & 14.1 & 9 & 31.03 & 4 & 8.7 \\
\hline $1-2$ & 15 & 20.0 & 1 & 25.00 & 14 & 19.7 & 11 & 37.93 & 4 & 8.7 \\
\hline $2-3$ & 17 & 22.7 & 0 & 0.00 & 16 & 22.5 & 9 & 31.03 & 7 & 15.2 \\
\hline $3-4$ & 5 & 6.7 & 0 & 0.00 & 6 & 8.5 & 0 & 0.00 & 5 & 10.9 \\
\hline $4-5$ & 11 & 14.7 & 0 & 0.00 & 11 & 15.5 & 0 & 0.00 & 11 & 23.9 \\
\hline $5-6$ & 15 & 20.0 & 0 & 0.00 & 14 & 19.7 & 0 & 0.00 & 15 & 32.6 \\
\hline \multicolumn{3}{|l|}{$\chi^{2}\left({ }^{\mathrm{MC}} p\right)$} & \multicolumn{4}{|c|}{$6.689(0.138)$} & \multicolumn{4}{|c|}{$40.360^{*}\left(<0.001^{*}\right)$} \\
\hline
\end{tabular}

$\chi^{2}$ : value for Chi square test

MC: Monte Carlo test

*: Statistically significant at $\mathrm{p} \leq 0.05$

$\mathrm{P}: \mathrm{p}$ value for comparing between the two studied groups

FE: Fisher Exact test

Site of ST segment elevation (STEMI) and its prevalence among H-FABP positive and H-FABP negative groups:-

- $\quad$ Our study showed that there was no statistically significant difference between the H-FABP positive and HFABP negative patients groups as regards the site of infarction. (Table 10)

- The most common site of infarction assessed by electrocardiogram in H-FABP positive patients is anterior wall STEMI $(55.9 \%)$, whereas the most common in H-FABP negative patients is lateral wall STEMI (42.9\%). (Table 10) 
Table 10:- Relation between H-FABP with site of infarction by ECG

\begin{tabular}{|c|c|c|c|c|c|c|}
\hline & \multicolumn{4}{|c|}{ H FABP } & \multirow[t]{3}{*}{$\chi^{2}$} & \multirow[t]{3}{*}{${ }^{F E_{p}}$} \\
\hline & \multicolumn{2}{|c|}{$\begin{array}{l}\text {-ve } \\
(n=7)\end{array}$} & \multicolumn{2}{|c|}{$\begin{array}{l}+v e \\
(n=68)\end{array}$} & & \\
\hline & No. & $\%$ & No. & $\%$ & & \\
\hline \multicolumn{7}{|l|}{ Site of infarction } \\
\hline Anterior STEMI & 2 & 28.6 & 38 & 55.9 & 0.042 & 1.000 \\
\hline Lateral STEMI & 3 & 42.9 & 2 & 2.9 & 8.258 & 0.041 \\
\hline Inferior STEMI & 2 & 28.6 & 28 & 41.2 & 0.127 & 1.000 \\
\hline$\chi^{2}=\left({ }^{\mathrm{MC}} \mathrm{p}\right)$ & \multicolumn{4}{|c|}{$15.274(0.072)$} & & \\
\hline
\end{tabular}

Echocardiography characteristics among the H-FABP positive and H-FABP negative groups:-

- There was a statistically significant difference as regard EF\% between the H-FABP positive and H-FABP negative patients, where H-FABP positive and negative patients had good LVEF percentage $(\mathrm{P}$ value $=<$ 0.001). (Table 11)

Table 11:- Relation between H-FABP with LVEF and WMSI

\begin{tabular}{|c|c|c|c|c|c|c|}
\hline & \multicolumn{4}{|c|}{ H-FABP } & \multirow{3}{*}{$\chi^{2}$} & \multirow[t]{3}{*}{${ }^{\mathrm{MC}} \mathbf{p}$} \\
\hline & \multicolumn{2}{|c|}{$\begin{array}{l}\text {-ve } \\
(n=7)\end{array}$} & \multicolumn{2}{|c|}{$\begin{array}{l}+\mathrm{ve} \\
(\mathrm{n}=68)\end{array}$} & & \\
\hline & No. & $\%$ & No. & $\%$ & & \\
\hline \multicolumn{7}{|l|}{ LVEF } \\
\hline$<40 \%$ & 0.0 & 0.0 & 11 & 16.2 & \multirow[t]{2}{*}{0.269} & \multirow[t]{2}{*}{$<0.001^{*}$} \\
\hline$>40 \%$ & 7.0 & 100.0 & 57 & 83.8 & & \\
\hline \multicolumn{7}{|l|}{ WMSI } \\
\hline Mean \pm SD & \multicolumn{2}{|c|}{$1.07 \pm 0.45$} & \multicolumn{2}{|c|}{$1.36 \pm 0.74$} & $\mathrm{t}=2.073^{*}$ & $0.042^{*}$ \\
\hline
\end{tabular}

$\chi^{2}$ : value for Chi square test

MC: Monte Carlo test

t: Student t-test

Differences between H-FABP and Troponin as regards sensitivity, specificity, Positive Predictive Value (PPV), Negative Predictive Value (NPV) and Accuracy:-

- There is a statistically significant difference between studied population as regards sensitivity, specificity, PPV, NPV and accuracy. (Table 12, 13)

- Sensitivity of H-FABP is significantly higher than troponin in $0-3 \mathrm{~h}$ from onset of chest pain $(92.86 \%$ versus $81.8 \%$ for troponin). (Table 12)

- Troponin has significantly higher sensitivity than H-FABP in 3-6 h of onset of chest pain $(91.49 \%$ versus $89.36 \%$ respectively). (Table $\mathbf{1 3}$ )

- H-FABP has significantly higher sensitivity than troponin in 0-1, 1-2 and 2-3h of onset of chest pain. (Tables 14, 15, 16)

Table 12:- Agreement (sensitivity, specificity and accuracy) for HFABP and troponin (0 - 3) hours.

\begin{tabular}{|c|c|c|c|c|c|c|c|c|}
\hline & & Control & Cases & : & & $\vec{a}$ & $\vec{z}$ & 冚 \\
\hline \multirow[t]{2}{*}{ HFABP } & -ve & 23 & 4 & \multirow[t]{2}{*}{92.86} & \multirow[t]{2}{*}{90.0} & \multirow[t]{2}{*}{92.86} & \multirow[t]{2}{*}{92.0} & \multirow[t]{2}{*}{92.45} \\
\hline & +ve & 2 & 40 & & & & & \\
\hline \multirow[t]{2}{*}{ Troponin } & -ve & 25 & 29 & \multirow[t]{2}{*}{81.8} & \multirow[t]{2}{*}{88.2} & \multirow[t]{2}{*}{81.8} & \multirow[t]{2}{*}{88.2} & \multirow[t]{2}{*}{85.7} \\
\hline & + ve & 0 & 15 & & & & & \\
\hline
\end{tabular}


Table 13: Agreement (sensitivity, specificity and accuracy) for HFABP and troponin (3 - 6) hours

\begin{tabular}{|c|c|c|c|c|c|c|c|c|}
\hline & & Control & Cases & : & 莺 & $\vec{a}$ & $\frac{z}{z}$ & 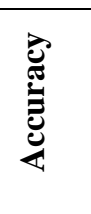 \\
\hline \multirow[t]{2}{*}{ HFABP } & -ve & 23 & 0 & \multirow[t]{2}{*}{89.36} & \multirow[t]{2}{*}{95.21} & \multirow[t]{2}{*}{92.89} & \multirow[t]{2}{*}{82.14} & \multirow[t]{2}{*}{90.28} \\
\hline & + ve & 2 & 31 & & & & & \\
\hline \multirow[t]{2}{*}{ Troponin } & -ve & 25 & 0 & \multirow[t]{2}{*}{91.49} & \multirow[t]{2}{*}{100.0} & \multirow[t]{2}{*}{100.0} & \multirow[t]{2}{*}{80.21} & \multirow[t]{2}{*}{94.44} \\
\hline & +ve & 0 & 31 & & & & & \\
\hline
\end{tabular}

Table 14:- Agreement (sensitivity, specificity and accuracy) for HFABP and troponin (0 - 1) hours

\begin{tabular}{|c|c|c|c|c|c|c|c|c|}
\hline & & Control & Cases & 㫄 & 莺 & $\vec{a}$ & $\vec{z}$ & 异 \\
\hline \multirow[t]{2}{*}{ HFABP } & -ve & 23 & 3 & \multirow[t]{2}{*}{83.33} & \multirow[t]{2}{*}{92.0} & \multirow[t]{2}{*}{83.33} & \multirow[t]{2}{*}{92.0} & \multirow[t]{2}{*}{89.19} \\
\hline & +ve & 2 & 10 & & & & & \\
\hline \multirow[t]{2}{*}{ Troponin } & -ve & 25 & 9 & \multirow[t]{2}{*}{81.0} & \multirow[t]{2}{*}{92.59} & \multirow[t]{2}{*}{80.8} & \multirow[t]{2}{*}{86.53} & \multirow[t]{2}{*}{87.79} \\
\hline & $+v e$ & 0 & 4 & & & & & \\
\hline
\end{tabular}

Table 15:- Agreement (sensitivity, specificity and accuracy) for H-FABP and troponin (1 - 2) hours.

\begin{tabular}{|c|c|c|c|c|c|c|c|c|}
\hline 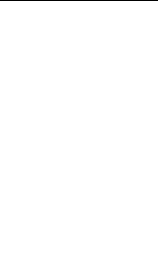 & & Control & Cases & : & 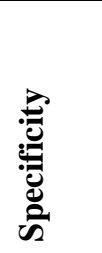 & $\vec{a}$ & $\frac{\vec{z}}{\mathbf{z}}$ & 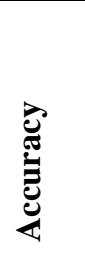 \\
\hline \multirow[t]{2}{*}{ HFABP } & -ve & 23 & 1 & \multirow[t]{2}{*}{88.24} & \multirow[t]{2}{*}{87.0} & \multirow[t]{2}{*}{98.0} & \multirow[t]{2}{*}{92.0} & \multirow[t]{2}{*}{95.0} \\
\hline & $+v e$ & 2 & 14 & & & & & \\
\hline \multirow[t]{2}{*}{ Troponin } & -ve & 25 & 11 & \multirow[t]{2}{*}{85.67} & \multirow[t]{2}{*}{86.21} & \multirow[t]{2}{*}{82.46} & \multirow[t]{2}{*}{89.44} & \multirow[t]{2}{*}{85.91} \\
\hline & + ve & 0 & 4 & & & & & \\
\hline
\end{tabular}

Table 16:- Agreement (sensitivity, specificity and accuracy) for HFABP and troponin (2 - 3) hours.

\begin{tabular}{|c|c|c|c|c|c|c|c|c|}
\hline & & Control & Cases & : & 矛 & $\vec{a}$ & $\frac{\vec{z}}{\mathbf{z}}$ & 冚 \\
\hline \multirow[t]{2}{*}{ HFABP } & -ve & 23 & 0 & \multirow[t]{2}{*}{88.89} & \multirow[t]{2}{*}{100.0} & \multirow[t]{2}{*}{100.0} & \multirow[t]{2}{*}{92.0} & \multirow[t]{2}{*}{92.86} \\
\hline & + ve & 2 & 16 & & & & & \\
\hline \multirow[t]{2}{*}{ Troponin } & -ve & 25 & 9 & \multirow[t]{2}{*}{85.29} & \multirow[t]{2}{*}{93.33} & \multirow[t]{2}{*}{84.55} & \multirow[t]{2}{*}{89.44} & \multirow[t]{2}{*}{85.70} \\
\hline & +ve & 0 & 7 & & & & & \\
\hline
\end{tabular}

\section{Statistical Analysis:-}

Data were fed to the computer and analyzed using IBM SPSS software package version 20.0. (Kirkpatrick LA and Feeney BC 2013) Qualitative data were described using number and percent. Quantitative data were described using range (minimum and maximum), mean, standard deviation and median. Comparison between different groups 
regarding categorical variables was tested using Chi-square test. When more than $20 \%$ of the cells have expected count less than 5, correction for chi-square was conducted using Fisher's Exact test or Monte Carlo correction. The distributions of quantitative variables were tested for normality. If it reveals normal data distribution, parametric tests was applied. If the data were abnormally distributed, non-parametric tests were used. For normally distributed data, comparisons between two independent populations were done using independent $t$-test. For normally distributed data F-test (ANOVA) were used to compare between the different studied groups. Correlations between two quantitative variables were assessed using Pearson or spearman coefficients regarding normality of the data. Significance test results are quoted as two-tailed probabilities. Significance of the obtained results was judged at the $5 \%$ level. (7)

\section{Discussion:-}

Management or excluding of patients with acute chest pain due to acute coronary syndrome (ACS) is a common and difficult challenge for emergency physicians. (8)

The diagnosis of cardiac ischemia remains a challenge in contemporary emergency medicine. On the other hand, unjustified referral of patients without ACS increases the workload in the emergency department and causes unnecessary load. So adequate diagnostic assessment of ACS patients leads to limited unnecessary referral of nonACS patients, but may be difficult to achieve. Therefore, a diagnostic strategy to rule in acute myocardial infarction on emergency department presentation is likely to be developed. (9)

Previously, the cardiac troponins remain the most widely used biomarkers for the diagnosis of ACS by virtue of their high sensitivity for minor myocardial injury, almost absolute specificity for myocardial damage, and most importantly, their ability to risk-stratify patients with suspected ACS. Their widespread recognition was supported by the inclusion of cardiac troponin as the preferred biomarker in the diagnosis of ACS both in the New Definition of MI (1999), further reinforced in the Universal Definition of Myocardial Infarction (2007) (10) and lately the third Universal Definition of Definition of Myocardial Infarction (2012). (11)

H-FABP has emerged as a promising early specific marker of myocardial injury; making it a suitable biomarker to be used in the ED in cases presented with cardiac chest pain. H-FABP is known to be released rapidly from the myocardium into the blood stream as early as 20 minutes following ischemic injury. Since H-FABP is a smaller molecule than myoglobin, cTnT, and CK-MB, it peaks earlier than these other molecules when there is cardiomyocyte damage. (12)

So lately, (H-FABP) was used as a rapid, qualitative "point of care" tests detecting (h-FABP) and recently quantitative tests have become also available. (13)

In the present study, the proportion of patients with positive (H-FABP) results was older than those with (H-FABP) negative results $(56.34 \pm 11.89$ vs. $55.43 \pm 10.01)$. Male gender was predominated in both (h-FABP) positive and negative group (69.1\% and 71.4 respectively). These findings were similar to other studies. (14)

Probably men have higher plasma H-FABP concentrations than women because of their larger muscle mass. HFABP is eliminated from the circulation predominantly by renal clearance and renal function decreases with age, so plasma H-FABP concentrations in this study were higher in old age group than young age group which agree with those published by Galán A et al. (15)

In addition systolic blood pressure was higher in patients with ACS-STEMI as compared to control group as well as in H-FABP positive patients rather than in H-FABP negative patients similar to Elmadbouh I. et al 2012. (16) Whereas the diastolic blood pressure was not significantly higher in patients with ACS as compared to control group may be due to that those patients belong to the category of only systolic hypertension in contrast to Santos et al. 2011 findings. (17)

Serum H-FABP levels were significantly elevated in diabetic patients when compared with that of control subjects. Hence Oxidative stress caused by hyperglycemia may provide an explanation for this association between diabetes and $\mathrm{CAD}$, as it causes endothelial dysfunction and increases prevalence of atherogenesis. (18) 
Also, serum total cholesterol, serum triglycerides and low density lipoproteins had higher levels opposite to high density lipoproteins which showed lower levels in patients with STEMI than control group ( $p$ value $<0.001$ ). These coincided with Spector R and Snapinn S 2011. (19) Furthermore the triglycerides showed positive correlation with increased risk for CAD. Several analyses indicate that triglycerides are independent risk factor for CAD, after adjustment for age and sex. Even though elevated levels of triglycerides in the blood are not independent risk factor in $\mathrm{CAD}$, there is an indirect association of triglycerides with atherosclerosis. (20)

The present study revealed that smoking is a highly risk factor of ACS-STEMI as it was higher but without significant statistical difference in cases as compared to the control group regarding, as well as in H-FABP positive rather than H-FABP negative patients. These results were in agreement with other studies like McCann CJ, Glover BM et al. 2009. (21)

In contrast to Mohammad AM, Sheikho SK et al. 2013 (22) a non-significant statistical difference between ACSSTEMI patients and controls regarding positive family history of premature CAD were seen in the present work. Before discharge from the hospital transthoracic echocardiography (TTE) was performed to all the study population. H-FABP positive patients had more poor LV systolic function compared to H-FABP negative patients. (23)

As regards mechanical complications; $4 \%$ patients had mechanical complications i.e. one of them had rupture anterior papillary muscle with severe mitral regurgitation and the others had ventricular septal rupture (basal septal rupture with inferior STEMI and mid-apical septal rupture with anterior STEMI). All of them belonged to H-FABP positive group of patients. The valuable biomarker related to early mechanical complications of AMI was CRP. High serum-CRP is related to early mechanical complications of AMI in support to Wang F, Pan W 2011. (24)

As regards acute setting and admission complications such as cardiogenic shock, our work showed that $5.3 \%$ of $\mathrm{H}-$ FABP positive, while no any of H-FABP negative patients had Killip class IV. In agreement to our study, Nurwahyudi 2014 (25) showed that H-FABP increased along with increase in Killip class and this is probably due to enhanced myocardial necrosis or ischemia in patients associated with higher Killip class. It was shown in our study that the concentration of H-FABP within 6 hours in AMI group was significantly higher than that in the control group, the same like Orak M, Ustündağ M et al. 2012. (26)

H-FABP is also a sensitive and specific marker for the early detection of AMI, especially within $3 \mathrm{~h}$, after the onset of chest pain (92.86\% and $90.0 \%$ versus $81.8 \%$ and $88.2 \%$ for Troponin), in addition the measurement of serum $\mathrm{H}$ FABP concentration has had high diagnostic accuracy for AMI in 0-3 h from onset of chest pain than troponin (92.45 versus 85.79 for Troponin) and negative predictive value in the early diagnosis of myocardial infarction (STEMI), as H-FABP negative predictive value was $92.0 \%$ compared to (cTn-T) which was $78.2 \%$. These match with Figiel T, Wraga M et al. 2011. (27) Whereas, in 3-6 hours of onset of chest pain; (cTn-T) had a higher sensitivity than H-FABP (91.49\% vs. 89.36\%) pertain with Chan CP, Sum KW et al. 2008. (28)

Interestingly this work showed false positive H-FABP results in some control subjects (NPV of 76.67\%). Skeletal muscle damage and strenuous exercise may result in the leakage of H-FABP, and this resulted in false positive tests that interfere with the results of the assays. This can explain the presence of 2 false positive persons in our study as published by Nagahara et al. 2006. (29)

Our work showed false negative H-FABP results in some case subjects i.e. 4 cases during 0-3 h of onset of chest pain. Levels of H-FABP are detectable as early as 2 to $3 \mathrm{~h}$ and typically return to baseline levels within 12 to $24 \mathrm{~h}$ of the initial insult similar to that of James A et al. 2007. (30)

Time lag between onset of myocardial necrosis and appearance of H-FABP in circulation is explained by: Firstly, release of heart fatty acid binding protein is not clear at present whether H-FABP reaches the circulation transendothelial or via the lymphatic system after its release from the cell into the intercellular space. The rapid appearance in blood may suggest the first route and delay explains lymphatic route. (31)

Secondarily, myocardial ischemia results in a significantly higher level of fatty acids in the plasma and the myocardial tissue, which can be harmful to the heart. The presence of H-FABP therefore may serve a protective function for the myocardial cells against the oxidation of these fatty acids while still having these substances readily 
available for the metabolic needs of the cell. During ischemia AMI, H-FABP leaks out of myocardial tissue and its concentration increases in plasma gradually. (32)

However, First, in ST-elevated ACS, biomarker tests are not valuable, since the diagnosis of infarction is based on the ST-elevations (33). Therefore, biomarker research in ACS is focusing on non ST elevated ACS. Another drawback of our study is the use of non-high sensitive, old-fashioned troponin tests, which makes the comparison with H-FABP irrelevant. High sensitive troponin is an earlier marker than old fashioned troponin and compared to high sensitive troponin, the value of H-FABP could disappear.

\section{Conclusion:-}

H-FABP biomarker test could be the earliest biomarker in patients with acute typical ischemic chest pain in the context of (STEMI), who came to the ED within 6 hours of chest pain onset. Our results supported the evidence that H-FABP was an early marker of ischemia; that was detected before any detectable change in (cTn-T) even in the absence of myocardial necrosis. This is especially useful to rule out ischemia in the ED, thereby making H-FABP a potentially useful biomarker for early detection of cardiac ischemia. H-FABP should prove its value when compared to, or assessed besides, high sensitive trop assays.

\section{References:-}

1. Chen L, Guo X and Yang F (2004): Role of Heart-Type Fatty Acid Binding Protein in Early Detection of Acute Myocardial Infarction in Comparison with cTnI, CK-MB and Myoglobin. Journal of Huazhong University of Science and Technology; 24: 449- 451.459.

2. Bassand JP, Hamm CW, Ardissino D, Boersma E, Budaj A, Fernández-Avilés F, Fox KA, Hasdai D, Ohman EM, Wallentin L, Wijns W. (2007): Guidelines for the diagnosis and treatment of non-ST-segment elevation acute coronary syndromes. Eur Heart J. 2007 Jul; 28(13):1598-660. Epub 2007 Jun 14.

3. Loria V, Leo M, Biasillo G, Dato I\& Biasucci LM (2008): Biomarkers in Acute Coronary Syndrome. Biomarker Insights; 5: 453-468.

4. Bruins Slot MH, van der Heijden GJ, Rutten FH, van der Spoel OP, Mast EG, Bredero AC, Doevendans PA, Glatz JF, Hoes AW (2008): Heart-type Fatty acid-binding protein in Acute Myocardial infarction Evaluation (FAME): background and design of a diagnostic study in primary care. BMC Cardiovasc Disord; 15: 8-14. 9

5. McMahon CG, Lamont JV, Curtin E, McConnell RI, Crockard M, Kurth MJ, Crean P, Fitzgerald SP (2011): Diagnostic accuracy of heart-type fatty acid-binding protein for the early diagnosis of acute myocardial infarction. Am J Emerg Med; 30(2):267-74.

6. Chandran PA, Wani BA, Satish OS, Mohammed N (2014): Diagnosis of Non-ST-Elevation Acute Coronary Syndrome by the Measurement of Heart-Type Fatty Acid Binding Protein in Serum: A Prospective Case Control Study. Journal of Biomarkers Volume 2014, Article ID 624930, 9 pages http://dx.doi.org/10.1155/2014/624930

7. Kirkpatrick LA and Feeney BC (2013): A simple guide to IBM SPSS statistics for version 20.0. Student ed. Belmont, Calif.: Wadsworth, Cengage Learning; x, 115 p.

8. Leslie E, Geoffrey J and James M (1991): Statistical analysis. In: Interpretation and uses of medical statistics ( $4^{\text {th }}$ edition). Oxford Scientific Publications (pub). Pp.411-6.

9. Hamm CW1, Bassand JP, Agewall S, Bax J, Boersma E, Bueno H, Caso P, Dudek D, Gielen S, Huber K, Ohman M, Petrie MC, Sonntag F, Uva MS, Storey RF, Wijns W, Zahger D; ESC Committee for Practice Guidelines. (2011): ESC Guidelines for the management of acute coronary syndromes in patients presenting without persistent ST-segment elevation: The Task Force for the management of acute coronary syndromes (ACS) in patients presenting without persistent ST-segment elevation of the European Society of Cardiology (ESC). Eur Heart J. 2011 Dec; 32(23):2999-3054. doi: 10.1093/eurheartj/ehr236. Epub 2011 Aug 26.

10. Madeleine P, Domanovits H and Fazelnia C (2008): Human heart-type fatty-acid-binding protein as a point-ofcare test in the early diagnosis of acute myocardial infarction. QJM; 100: 203-210.

11. Thygesen K; Alpert JS and White HD; (2007): Universal Definition of Myocardial Infarction. The European Society of Cardiology, the American College of Cardiology Foundation, the American Heart Association, and the World Heart Federation. DOI: 10.1161/CIRCULATIONAHA.107.187397

12. Thygesen K; Alpert JS and Jaffe AS, (2012): Third Universal Definition of Myocardial Infarction. The European Society of Cardiology, American College of Cardiology Foundation, American Heart Association, Inc., and the World Heart Federation. DOI: 10.1161/CIR.0b013e31826e1058 
13. Pasaoglu H, Ofluoglu E, Ilhan M.N, Cengel A, Ozdemir M, Durakoglugil E \& Erden M (2007): The Role of Heart-Type Fatty Acid-Binding Protein (H-FABP) in Acute Myocardial Infarction (AMI) Compared to Conventional Cardiac Biochemical Markers. Turk J Med Sci .37: 61-67.

14. Bruins Slot M.H.E., Rutten F.H., van der Heijden G.J.M.G., Doevendans PA, Mast EG, Bredero AC, van der Spoel OP, Glatz JF, Hoes AW. (2013): Diagnostic value of a heart-type fatty acid-binding protein (H-FABP) bedside test in suspected acute coronary syndrome in primary care. International Journal of Cardiology 168 (2013) 1485-1489

15. Niizeki T1, Takeishi Y, Arimoto T, Takabatake N, Nozaki N, Hirono O, Watanabe T, Nitobe J, Harada M, Suzuki S, Koyama Y, Kitahara T, Sasaki T, Kubota I (2007): Heart-type fatty acid-binding protein is more sensitive than troponin $\mathrm{T}$ to detect the ongoing myocardial damage in chronic heart failure patients. J Card Fail; 13(2): 120-7.

16. Galán A1, Curós A, Valle V. (2010): Biomarkers for detection and prediction in acute coronary syndrome. Med Clin (Barc). 2010 Apr 17; 134(11):499-504. doi: 10.1016/j.medcli.2009.01.036. Epub 2009 May 21.

17. Elmadbouh I., Mahfouz R., Bayomy N., Faried W., Ghanayem N. (2012): The value of human heart-type fatty acid binding protein in diagnosis of patients with acute chest pain. The Egyptian Heart Journal, Volume 64, Issue 4, December 2012, Pages 179-184

18. Santos ES, Baltar VT, Pereira MP, Minuzzo L, Timerman A \& Avezum A (2011): Comparison between Cardiac Troponin I and CK-MB Mass in Acute Coronary Syndrome without ST Elevation. Arq Bras Cardiol. 2011 Mar; 96(3):179-87. Epub 2011 Feb 4.

19. Matheus AS, Tannus LR, Cobas RA, Palma SC, Negrato AC, 2 and Gomes BM (2013): Impact of Diabetes on Cardiovascular Disease: An Update. International Journal of Hypertension Volume 2013, Article ID 653789, 15 pages http://dx.doi.org/10.1155/2013/653789

20. Spector R and Snapinn S (2011): Statins for secondary prevention of cardiovascular disease: the right dose. Pharmacology.87: 63-9.

21. Sacks DB, Arnold M, Bakris GL, Bruns DE, Horvath AR, Kirkman MS, Lernmark A, Metzger BE, Nathan DM; National Academy of Clinical Biochemistry; Evidence-Based Laboratory Medicine Committee of the American Association for Clinical Chemistry. (2011): Guidelines and recommendations for laboratory analysis in the diagnosis and management of diabetes mellitus. DIABETES CARE, VOLUME 34, JUNE 2011

22. McCann CJ, Glover BM, Menown IB, Moore MJ, McEneny J, Owens CG, Smith B, Sharpe PC, Young IS \& Adgey JA (2009): Prognostic value of a multimarker approach for patients presenting to hospital with acute chest pain. Am J Cardiol. 103:22-8.

23. Mohammad AM, Sheikho SK, Tayib JM (2013): Relation of Cardiovascular Risk Factors with Coronary Angiographic Findings in Iraqi Patients with Ischemic Heart Disease. American Journal of Cardiovascular Disease Research, 2013, Vol. 1, No. 1, 25-29

24. Boscheri A, Wunderlich C, Langer M, Schoen S, Wiedemann B, Stolte D, Elmer G, Barthel P, Strasser RH (2010): Correlation of heart-type fatty acid-binding protein with mortality and echocardiographic data in patients with pulmonary embolism at intermediate risk. Am Heart J. 2010 Aug; 160(2):294-300. doi: 10.1016/j.ahj.2010.05.010.

25. Wang F, Pan W, Pan S, Wang S, Ge Q, Ge J. (2011): Usefulness of N-terminal pro-brain natriuretic peptide and C-reactive protein to predict ICU mortality in unselected medical ICU patients: a prospective, observational study. Crit Care. 2011; 15(1):R42.Nurwahyudi M. (2014): Correlation between Serum Level of H-FABP and (NT-proBNP) on Acute Coronary Syndrome Patient. Folia Medica Indonesiana Vol. 50 No. 2 April - June 2014 : 73-80

26. Orak M, Ustündağ M, Güloğlu C, Ozhasenekler A, Alyan O, Kale E. (2010): The role of the heart-type fatty acid binding protein in the early diagnosis of acute coronary syndrome and its comparison with troponin I and creatine kinase-MB isoform. Am J Emerg Med. 2010 Oct; 28(8):891-6. doi: 10.1016/j.ajem.2009.05.012. Epub $2010 \mathrm{Feb} 25$.

27. Figiel L, Małgorzata Wraga, Zbigniew Bednarkiewicz, Piotr Lipiec, Janusz Śmigielski (2011): Direct comparison of the diagnostic value of point-of-care tests detecting heart-type fatty acid binding protein or glycogen phosphorylase isoenzyme BB in patients with acute coronary syndromes with persistent ST-segment elevation. Kardiologia Polska; 69, 1: 1-6ISSN 0022-9032.

28. Chan CP , Sum KW , Cheung KY, Glatz JF , Sanderson JE, Hempet A, Lehmann M , Renneberg I \&Renneberg R (2008): Development of a quantitative lateral-flow assay for rapid detection of fatty acidbinding protein. J Immuno Methods; 279: 91-100. 
29. Nagahara D., Nakata T., Hashimoto A., Takahashi T, Kyuma M, Hase M, Tsuchihashi K\& Shimamoto K (2006): Early positive biomarker in relation to myocardial necrosis and impaired fatty acid metabolism in patients presenting with acute chest pain at an emergency room. Circ. J. 70:419-25.

30. James SK, Lindahl B, Armstrong P, Califf R, Simoons ML, Venge P\& Wallentin L (2007): A rapid troponin I assay is not optimal for determination of troponin status and prediction of subsequent cardiac events at suspicion of unstable coronary syndromes. Int J Cardiol. 93:113-20.

31. Gilardi E, Iacomini P, Marsiliani D, De Marco G, Covino M (2014): Biomarkers in the prediction and management of acute coronary syndromes: current perspectives. DOI http://dx.doi.org/10.2147/RRCC.S36294. Research Reports in Clinical Cardiology 2014:5 21-31

32. Viswanathan K, Kilcullen N, Morrell C, Thistlethwaite S.J, Sivananthan M.U, Hassan T.B, Barth J.H \& Hall A.S (2010): Heart-Type Fatty Acid-Binding Protein Predicts Long-Term Mortality and Re-Infarction in Consecutive Patients With Suspected Acute Coronary Syndrome Who Are Troponin-Negative. J Am Coll Cardiol. 55:2590-2598.

33. Thygesen K. and Searle J. (2013): Update on the Universal Definition of Acute Myocardial Infarction in the Light of New Data. Conference Papers in Medicine Volume 2013 (2013) 